\title{
Microstrip Antennas and Arrays on Photonic Band Gap Substrates
}

\author{
T Sudha and T S Vedavathy
}

\begin{abstract}
Patch antennas have some Limitations such as restricted band -width of operation, low gain and a potential decrease in radiation efficiency due to surface wave losses. In this paper, the ability of a Photonic Band Gap (PBG) substrate to minimize the surface wave effects is analyzed for a thick and high $\varepsilon_{\mathrm{r}}$ substrate. Two different types of PBGs are used, one the conventional dielectric PBG and the other a metallodielectric PBG. The PBG patch antenna showed significantly reduced levels of surface modes compared to conventional patch antenna, thus improving the gain and far- field radiation pattern. The results also showed that introducing metallic cylinders in dielectric crystals is more effective in suppressing side lobe radiation. The performance improvement of a twoelementarray of patches on the PBG substrate is also analyzed.
\end{abstract}

Index Terms - Photanic Band Gap, metallodielectric, microstrip antenna, surface waves

\section{INTRODUCTION}

A common property of most microstrip antennas is that the antenna element launches surface wave modes, in addition to the fields radiated into the space. The excitation of the surface waves lowers the overall radiation efficiency of the antenna. For finite-size substrates, the surface power will diffract from the edges of the substrates, resulting in a disturbance of the radiation pattern. The excitation of the surface waves also results in increased mutual coupling between distant antenna elements. For these reasons the excitation of surface Waves is generally undesirable. Microstrip antennas fabricated on a substrate with high $\boldsymbol{\varepsilon}_{\mathrm{r}}$ are strongly preferred for easy integration with MMIC RF frontend circuitry. However it is well known that patch antennas on high $\varepsilon_{\mathrm{r}}$ substrates are highly inefficient radiators due to surface wave losses and have very narrow frequency bandwidths. Suppression or reduction of surface waves improves antenna efficiency and reduces side lobe level that is caused by the diffraction of surface waves at the edges of the antenna substrates. Surface diffraction plays a major role when thick substrates are used to improve the bandwidth of the antenna. Power losses due to surface waves are as high as $60 \%$ of the radiated power when thick substrates with high $\varepsilon_{r}$ are used [1]. Photonic crystals (PC) can offer a real solution to this problem [2]-[4]. Utilized in patch antenna configurations as substrates, PCs suppress both substrates

Manuscript. received on February 02,2001

The authors are with ECE Dept., Indian Institute of Science, Bangalore,

India.e-mail: sudha@protocol.ece.iisc.ernet.in, veda@ece.iisc.ernet.in. modes and surface waves. PCs are a class of periodic metallic, dielectric or composite structures that exhibit pass and stop bands in their frequency response. Because of this they offer the property to forbid the propagation of the electromagnetic waves whose frequency is included within their stop hand-so called Photonic Band Gap (PBG). Frequency Band gap depends on the dielectric contrast between the atoms and host material, their dimensions, the periodicity and incidence angle of em waves. From largescale study made regarding the influence of the lattice shape, size and shape of the atoms, large circular holes on a triangular lattice found to give a large PBG, common for both TE and TM polarization. The triangular lattice is chosen because it has proved capable, in the simpler case of a 2-D PBG, of providing a stop band for any in plane $\left(\beta_{\mathrm{z}}=0\right)$ direction of propagation due to the high symmetry of the Brillouin zone (BZ) associated to it. PCs can be doped by introducing lattice defects into the structure. Thus if the periodicity in a PC is perturbed (by either removing or adding a material with a different dielectric constant) creating a 'defect', that is a state is created in the forbidden gap where an electromagnetic mode is allowed and localization of the energy occurs [5]. So if the substrate is periodically loaded to create a PBG crystal in such a way that the frequency of the substrate mode overlaps the stop band frequency of the crystal, the excited substrate mode exponentially decays thus reducing the energy lost into the substrate. Thus an increased amount of radiated power couples to space waves and this mechanism has the effect of reshaping the antenna pattern. [3], [4]. While a PC structure, such as a substrate with holes, could be thought of as a non-isotropic medium, the anisotropy is introduced into the equations through the positional dependence of the $\varepsilon_{\mathrm{r}}$, i.e. $D(r)=\varepsilon(\mathrm{r}) \mathrm{E}(\mathrm{r})$.

The Brillouin zone for the triangular lattice is a hexagon (Fig.1).

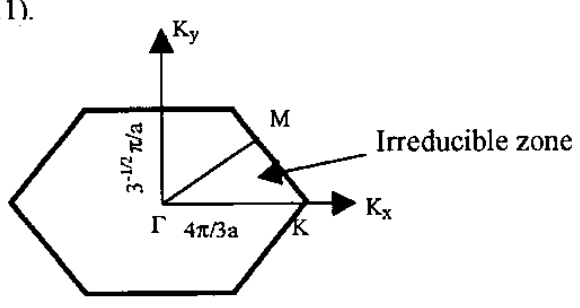

Fig.1 Brillouin Zone of

Triangular lattice 
A hand gap in the dispersion curves will show up at the BZ boundary, $\beta, a=\pi$.

Conventional PCs are made of two different dielectric materials, arrayed in a periodic lattice. Wide crystal variety can be added to existing structures by introducing both dielectric and metal components in a unit cell thus making them metallodielectric photonic crystals. A theoretical study of a PBF structure in 2-D metallodielectric PC that consists of square arrays of dielectric and perfectly conducting cylinders is reported in [6]. It is showed that introducing metallic cylinders in dielectric crystals can generate PBG, even when the dielectric PC exhibit no full band gap. Metallic implants also helps to have surface wave mode elimination in all directions [7]. Because of its large imaginary dielectric constant metallic core reflects radiation very efficiently at least at microwave and millimeter-wave frequencies, if it is surrounded by loss less dielectric. This enhances the rejection in the stop hand [8]. One of the best features of microstrip antennas is the ease with which they can he formed into arrays and a wide variety of arrays have been designed using microstrip elements. It is clear that PBG structures offer promising potcntial applications in array configurations, increasing the antenna efficiency together with the suppression of its mutual coupling through the substrate material. Reduced mutual coupling might provide a practical solution to the "scan blindness" problem. In this work, the improvements in the radiation characteristics are studied both for a single antenna and a 2-element array, made on a $50 \mathrm{mil}$ thick substrate with $\varepsilon_{t}$ of 10.2 and loss tangent 0.0023 loaded with a 2D triangular lattice of cylinders. With the conventional dielectric PC here a two dimensional metallodielectric PC is also used to obtain the hand gap. An FEM based software package HP-HFSS was used to analyze the antenna. The results showed reduced surface wave propagation along the PBG substrate resulting in improved gain and an increase in bandwidth. It was also established that introducing metallic cylinders in dielectric crystals is more effective in suppressing side lobe radiation.

\section{iI. ANTENNA DESIGN \& ANALYSIS}

A coaxial fed rectangular patch antenna was designed to work at $9 \mathrm{GHz}$. The grounded dielectric slab used was $50 \mathrm{mil}$ thick and having $\varepsilon_{\boldsymbol{T}}$ 10.2.At this frequency the higher order modes are below cut-off. According to Wood's criteria $\mathrm{h} / \lambda_{0}<0.023$ if the antenna is to launch no more than $25 \%$ of the total radiated power as surface waves. Since $\mathrm{TM}_{\infty}$ mode has no cut-off it should be present in all the substrates and its effect is more at high frequencies. In order to prohibit the propagation of this mode the substrate here is converted as a PBG structure by drilling triangular lattice of air holes in it. The lattice constant (a) is selected as $1.38 \mathrm{~cm}$, which is already proved to give a gap at approximately $9 \mathrm{GHz}[2]$. The radius of the holes is selected such that $\mathrm{r} / \mathrm{a}=0.48$ for getting maximum gap. The substrate size required to accommodate at least three periods of the structure was $7 \mathrm{x} 9 \mathrm{~cm}$ (fig.2).

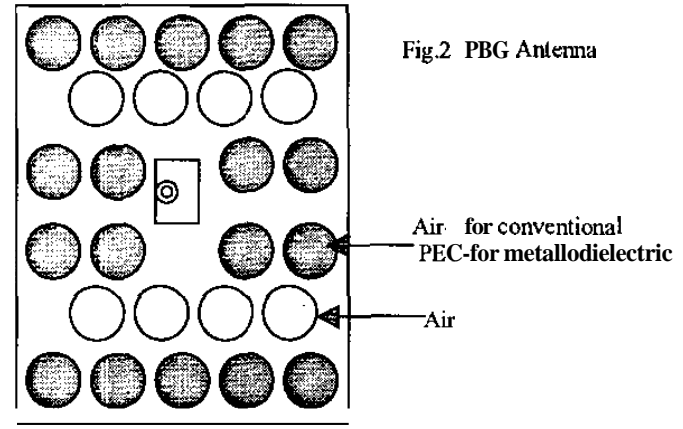

This substrate is later converted as a metallodielectric PBG structure by introducing triangular array of air and perfectly conducting cylinders (PEC) in it. The array geometry consists of two rectangular patch elements with co-axial feed on a finite grounded substrate. The patch elements are excited with opposite feed locations. The radiation characteristics of this antenna on a $2.1 \lambda_{0} \times 2.2 \lambda_{0}$ ground plane at $9 \mathrm{GHz}$ for an element spacing, $s=\lambda_{0} / 2$ is also analyzed on the PBG substrate. The analysis are done with PBG structures consisting of air holes only and those having air and perfectly conducting cylinders, with all the dimensions of the structures exactly the same. The results show introducing metallic cylinders in dielectric crystals is more effective in suppressing side lobe radiation than a structure having only dielectrics. For the performance comparison the single and array antennas are also analyzed on a normal substrate without PBG, with all the dimensions same. HFSS- a fullwave em simulator based on FEM in association with proper absorbing boundary conditions was used to analyze the structure. Convergence and minimization of errors was obtained by ensuring that enough iterations were used.

\section{Simulation Results}

\section{A. Single antenna}

Fig. 3 shows the return loss (sl1) of the PBG and reference patch for $\varepsilon_{r}=10.2$

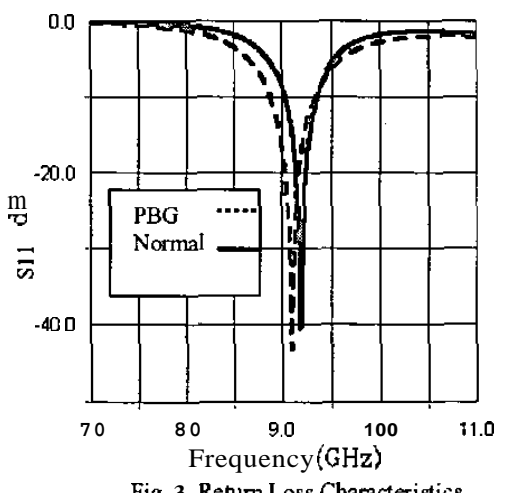

Fig 3 Retum Loss Characteristics 

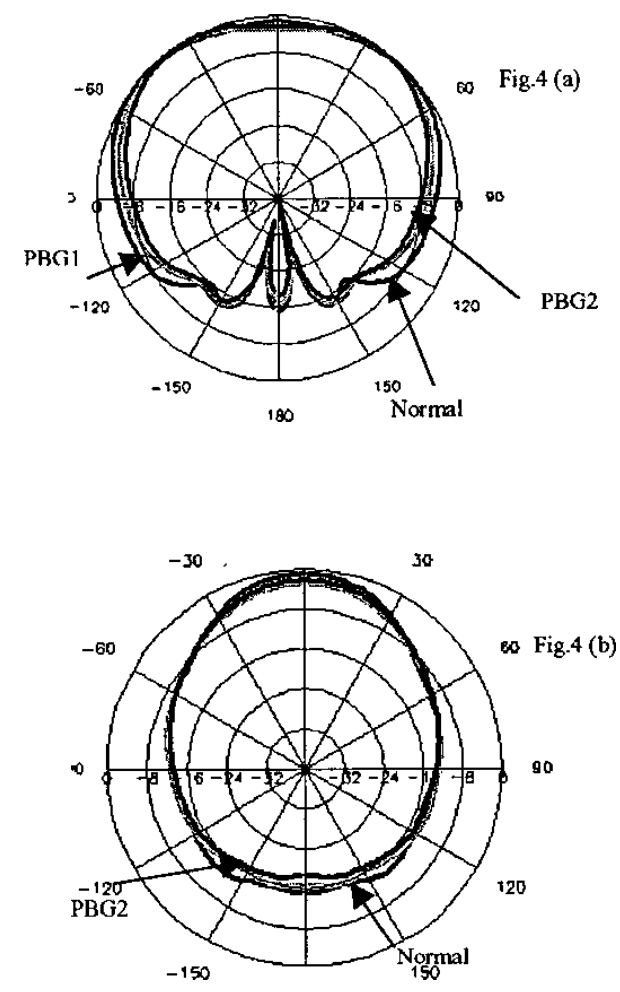

\begin{tabular}{|c|c|l|c|}
\hline \multirow{2}{*}{$\begin{array}{c}\text { Antenna } \\
\text { Parameters }\end{array}$} & \multirow{2}{*}{$\begin{array}{c}\text { Normal } \\
\text { Ant }\end{array}$} & \multicolumn{2}{|c|}{ PBG Antenna } \\
\cline { 3 - 4 } & & Air & Metello-dielectric \\
\hline Directivity (dB) & 5.48 & 6.54 & 6.94 \\
Gain (dB) & 4.88 & 6.37 & 6.88 \\
$\left|E_{0} \max \right|(\mathrm{v})$ & 13.58 & 16.12 & 17.04 \\
$\mid$ E $\max \mid(\mathrm{v})$ & 0.012 & 0 & 0 \\
B-W (\%) & 3.65 & 8.24 & 5.39 \\
\hline
\end{tabular}

antennas

1
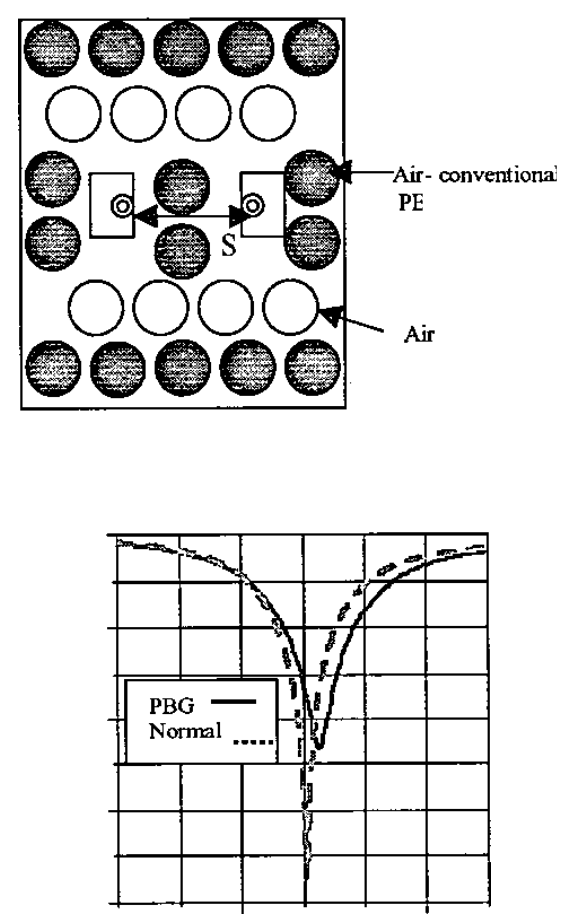

From Fig.6 it is seen that the $10 \mathrm{~dB}$ bandwidth is improved from $2.72 \%$ to $3.64 \%$. The radiation pattern of the array is shown in Fig.7 (a) and Fig.7 (b). With air PBG the antenna showed a side lobe reduction of $2 \mathrm{~dB}$ and a back lobe reduction of $3 \mathrm{~dB}$. With metallodielectric PBG the hack radiation remained the same but the side lobes showed a $6 \mathrm{~dB}$ reduction. There is a directivity and gain improvement of greater than $1 \mathrm{~dB}$. With bigger ground planes the back lobe level can be further improved, but the side lobe remains at 


\section{ConClusion}
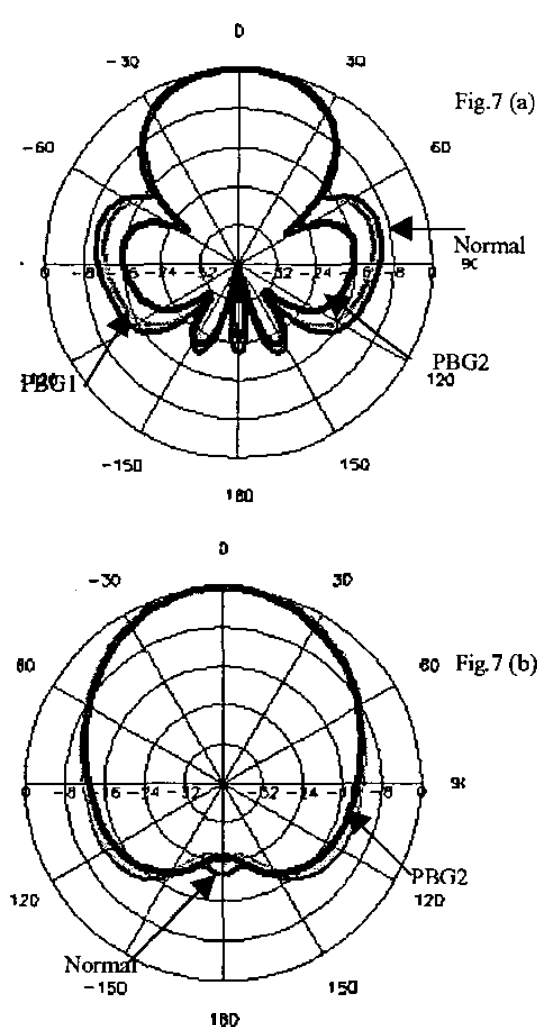

Fig.7. Comparison of radiation pattems of the array (a) E-Planepatterns (b) H-Plane patterns

TABLE II

COMPARISONOF ANTENNA ARRAY PARAMETERS

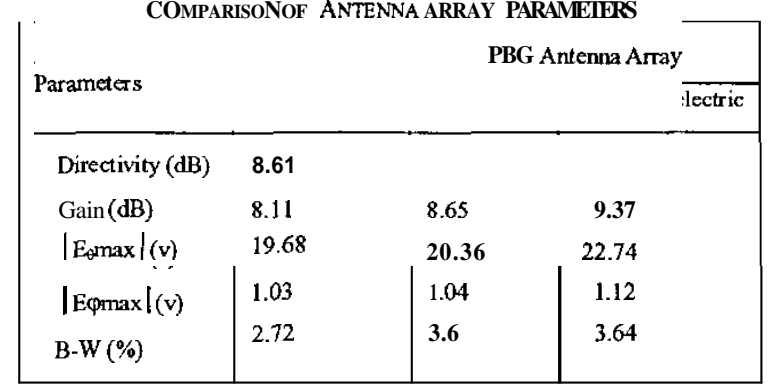

It is $\sec$ that PBG thus helps to reducc all the unwanted effects of surface waves and at the same time improved the directivity and gain of the antenna.
Comparisons between the results from a conventional patch antenna to a patch antenna on a PBG substrate show that reduction in the surface mode level is remarkable. This leads to an increase in the antenna gain and efficiency. The back radiation is also considerably reduced. The use of $P B G$ is advantageous for integrating antenna on high 4 substrates without losing performance. It also reduces unwanted coupling between nearby systems since it reduces back radiation and surface waves along antenna substrate. The results show introducing metallic cylinders in dielectric crystals is more effective in suppressing side lobe radiation than a structure having only dielectrics. Future applications in array configurations are under study for beam steering and gain enhancement.

\section{ACKNOWLEDGMENT}

The authors are grateful to HP for providing easy access to the software.

\section{REFERENCES}

[I] J. Bahl and P. Bhartia, Microstrip Antennas. Dedham, MA: Artech House, 1980.

[2] J. D. Jeannopoulos, R D. Meale, J. N. Winn, Photonic Crystals: Modeling the flow of Light, Princeton University Press, New Jercey, 1995

[31 Y. Qian, R. Coccioli, D. Sievenpiper, V. Radisic, E. Yablonovitch, T Itoh, "Microstrip patch antenna using novel PBG structures" Microwave Journal. Vol. 42, No. 1, pp. 66-76,Jan 1999

[4] Ramon Gonzalo, Peter de Maagt, Mario Sorrolo, "Enhanced Patch antenna performance by suppressing surface waves using PBG substrates," IEEE trans. Microuque Theory \& Tech.. Vol. 47, No. 11, pp. 2131-2138, Nov.1999

[S] Robert D. Meade, Karl D. Brommer, J. D. Joannopoulos, "Existence of a photonic band gap in two dimensions," Appl. Phys. Lett. Vol, .61, No.4, pp 495-497,July 1992

[6] Tosthio Suzuki and Paul K L.Yu,"Existence of photonic band gaps in two dimensional metallodielectric photonic crystals," Electromagnetics, pp. 321-335, 1999.

[7] Dan Sievenpiper and Eli Yablonovitch,"Eliminating surface currents with metallodielectric photonic. crystals," IEEE MTT-S digest,Baltimore, June 1998

181 E.R.Brown and O.B.Mcmahon,"Large electromagnetic slop bands in metallodielectric photonic crystals," Appl. Phys. lett., Vol. 67, No. IS, Pp. 2138+2140, Od. 1995

[9] B. Robert, C. Terret, J. P. Daniel, P. Pribetich, P. Kerinis, "Fundamental surface wave effects on microstrip antenna radiation," Electronic Letters, Val. 21, pp. $1112-1114,1985$ 